\title{
Intensive care unit electroencephalogram patterns
}

\author{
Walter Duarte MD, Victor Montalvan MD
}

The background frequencies of the EEG are one of the important aspects that helps describe the EEG patterns. EEG frequencies are measured in Hertz $(\mathrm{Hz})$, and there are 4 types: beta, alpha, theta, delta.

How to recognize beta, alpha, theta, and delta frequencies?

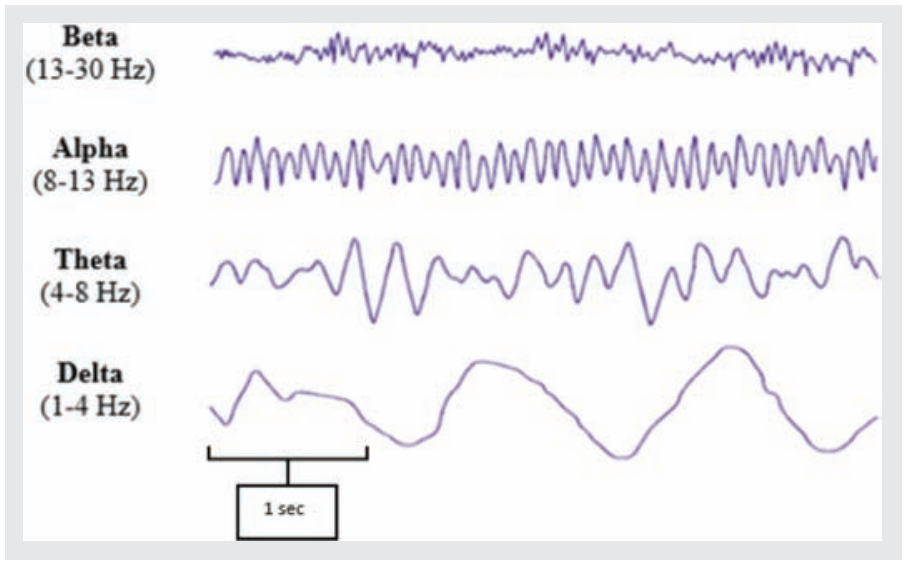

\section{Normal EEG}

\subsection{Normal aWAKE EEG}

It is characterized by the alpha rhythm $(8-13 \mathrm{~Hz})$ in the posterior regions of the head, also called posterior dominant rhythm. The alpha rhythm usually appears with eyes closed when the patient is relaxed, and attenuates with eyes open. Note the appearance of the alpha waves (arrow) after the patient closes his eyes and the attenuation when he starts counting. Note the muscle artifact (arrowhead) in the left temporal region.

Corresponding author: Walter Duarte Contact Information: Walter.Duarte@ttuhsc.edu DOI: $10.12746 /$ swrccc.v8i36.771

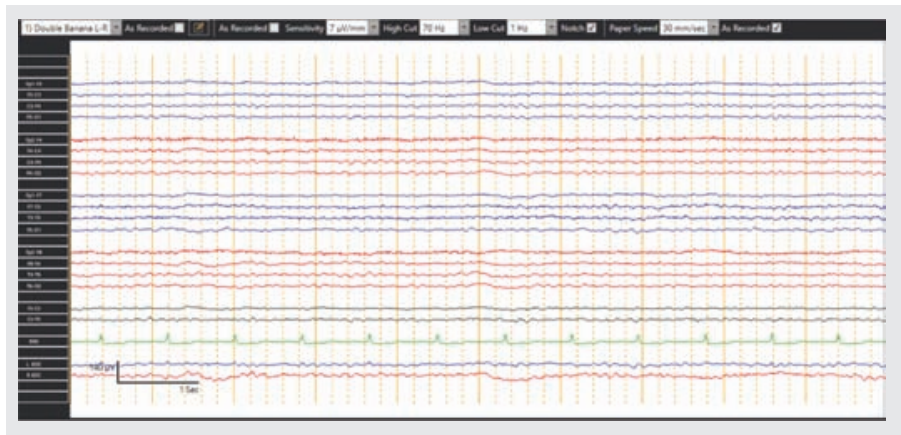

\subsection{Normal sLeEP EEG}

Different frequencies and wave forms can be seen in the EEG, depending on the stage of sleep, such as vertex waves (central channels), posterior occipital sharp transient of sleep (occipital channels), spindles and K-complexes (central channels), or slower frequencies. 


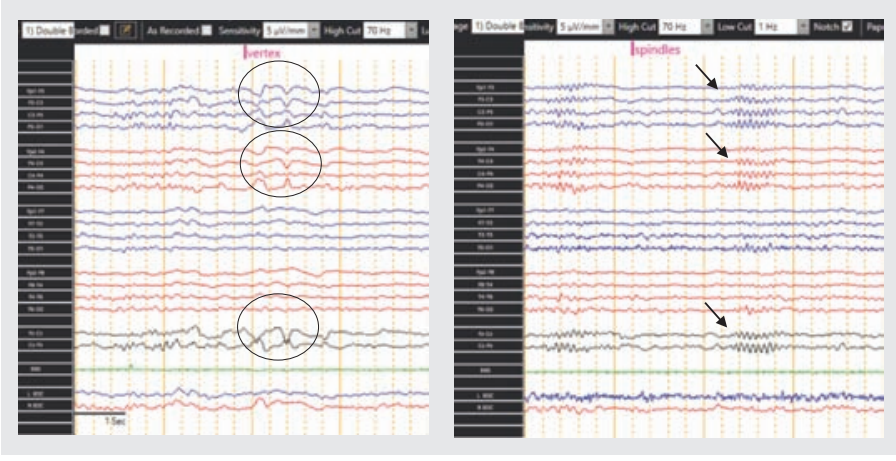

\section{ENCEPHALOPATHY}

This pattern is characterized by slow frequencies such as delta and theta waves. It denotes non-specific cerebral dysfunction. Encephalopathy can be graded from mild to profound. This pattern can be seen in multiple settings, including sedation, metabolic derangements, anoxic brain injury, etc.

\subsection{Mild encephalopathy}

There is absence of posterior dominant rhythm. Most of the background shows the presence of theta waves more than delta waves.

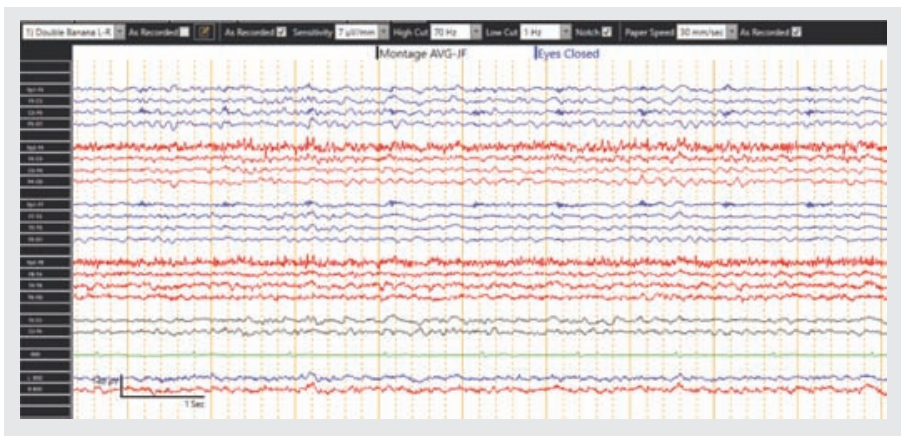

The EEG was recorded in a 73-year-old man with confusion, sepsis, and acute kidney injury.

\subsection{Moderate encephalopathy}

There is absence of posterior dominant rhythm. Most of the background shows the presence of delta (arrow) more than theta waves.

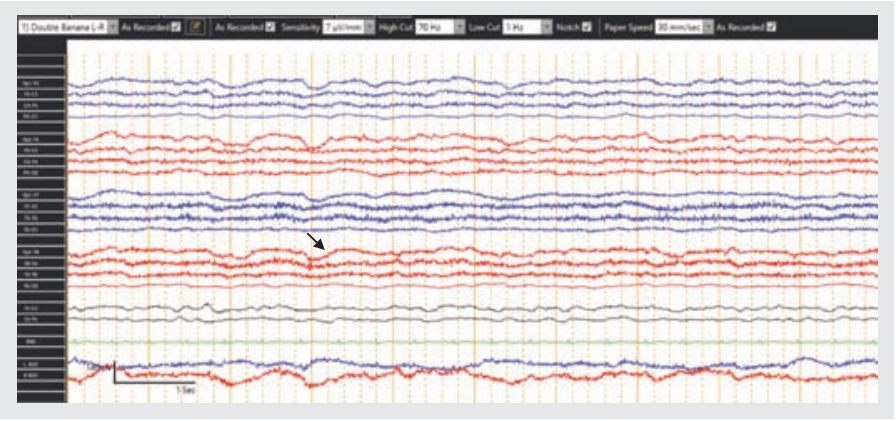

The EEG was recorded in a 26-year-old man with a history of seizures. Patient received sedatives in the emergency room.

\subsection{SeVERE encephalopathy}

Very slow frequencies, sometimes accompanied by simple generalized periodic discharges (including triphasic waves). The three components of the triphasic waves (arrow) are: the sharpest wave, followed by the tallest wave (downward deflection), and then the longest wave. A phase lag is often seen in this pattern (arrow head) which means that the anterior channels usually manifest the waves a few milliseconds before the posterior regions.

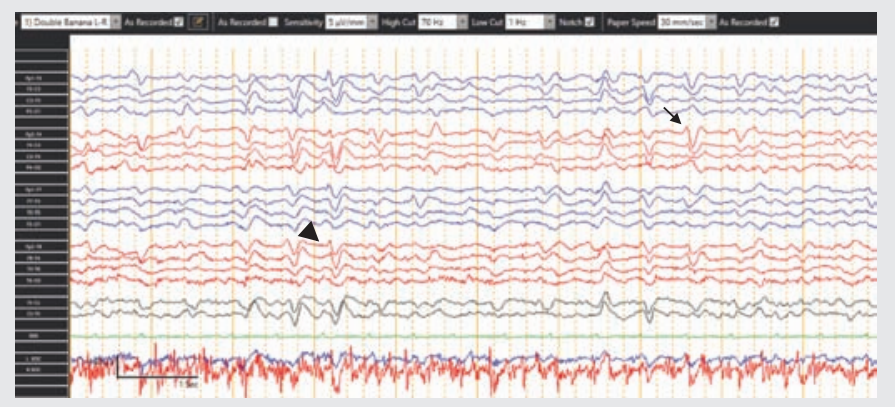

The EEG was recorded in a 74-year-old man with encephalopathy, responsive only to painful stimuli. Note the bisynchronous and symmetric triphasic waves.

\subsection{Profound suppression of the RHYTHM}

A suppressed EEG is defined as amplitudes less than 10 micro Volts. Note the absence of potentials 
when EEG is reviewed even at higher sensitivities (3 $\mathrm{microV} / \mathrm{mm}$ ).

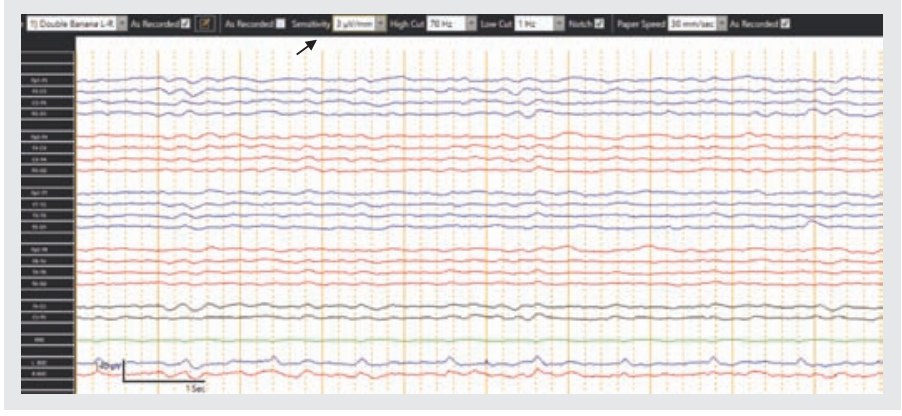

This electroencephalogram was recorded in a 72year-old man with altered mental status and respiratory failure. Patient was intubated and comatose.

\section{BURST-SUPPRESSION PATTERN}

Burst-suppression is an abnormal pattern in the electroencephalogram in which bursts of high-voltage slow waves and sharp waves alternate with periods of depressed background activity. Caution must be taken in consideration when interpreting this type of patterns since it can be seen in anoxic brain injury, prolonged status epilepticus, myoclonic status epilepticus, and deeply sedated patients.

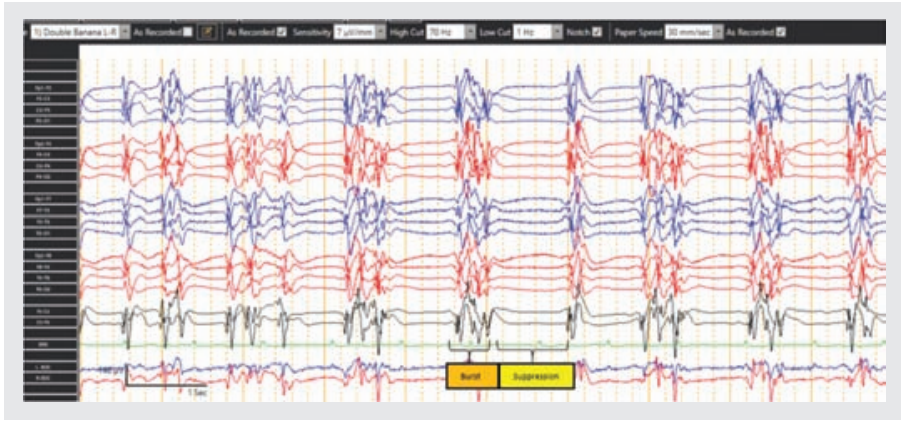

The EEG was recorded on a 60-year-old woman who had a cardiac arrest and was coded for approximately 10 minutes.

\section{FocAl SLOWING: LEFT HEMISPHERE SLOWING}

It is characterized by slow frequencies such as delta and theta waves in one specific region of the brain. It denotes focal non-specific cerebral dysfunction. Note the delta and theta waves over the left hemisphere (blue channels and arrows).

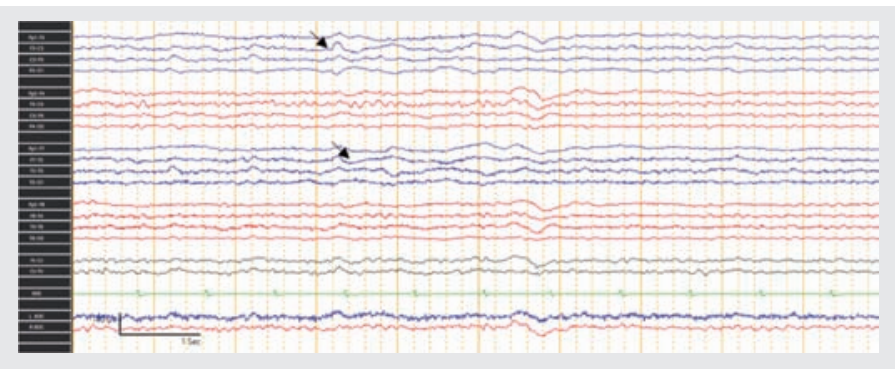

The electroencephalogram was recorded in a 67 -year-old man with left middle cerebral artery stroke.

\section{SubClinical status EPILEPTICUS}

This is a very common entity in critically ill patients. The EEG shows electrographic seizures without prominent motor symptoms.

Note the ictal discharges from the right frontotemporal region (red channels): faster activities, sharply contoured waves, more pronounced in F8-T4 and T4-T6 (arrow), and intermittent changes in frequency and amplitude.

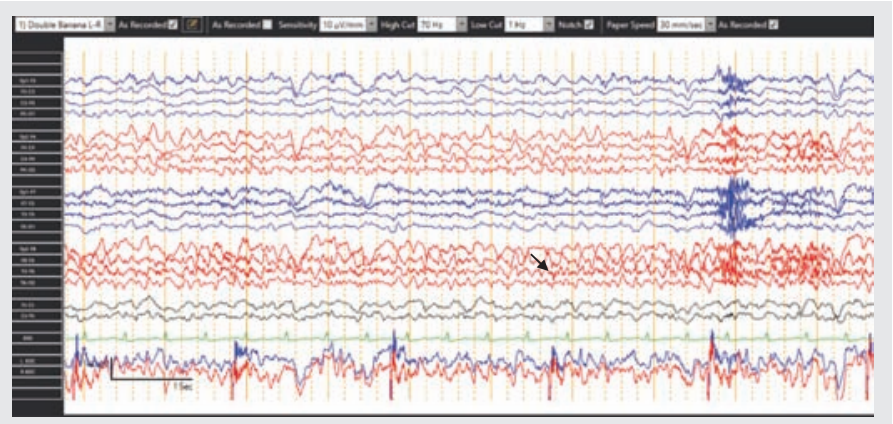

28-year-old woman with multiple and prolonged episodes of behavioral arrest.

Keywords: EEG, wave patterns, encephalopathy, burst-suppression 
Article citation: Duarte W, Montalvan V. Intensive care unit electroencephalogram patterns. The Southwest Respiratory and Critical Care Chronicles 2020;8(36):86-89 From: Department of Neurology, Texas Tech University Health Sciences Center, Lubbock, Texas

Submitted: $4 / 17 / 2020$

Accepted: $10 / 10 / 2020$

Reviewer: Jannatul Ferdous MD

Conflicts of interest: none

This work is licensed under a Creative Commons Attribution-ShareAlike 4.0 International License.

\section{REFERENCES}

1. Marcuse LV, Fields MC, Yoo J. Rowan's Primer of EEG. $2^{\text {nd }}$ edition. New York: Elsevier 2016.

2. LaRoche SM, Haider HA. Handbook of ICU EEG monitoring. $2^{\text {nd }}$ edition. New York: Springer Publishing Company 2018.

3. Stern J. Atlas of EEG Patterns. $2^{\text {nd }}$ edition. Philadelphia: Lippincott Williams \& Wilkins 2013. 